\title{
THE PREVALENCE OF RECURRENT APHTHOUS STOMATITIS IN PATIENTS WITH HEMATINIC DEFICIENCY
}

Meltem Koray ${ }^{1}$, Duygu Ofluoglu², Sacide Erden ${ }^{3}$, Alp Saruhanoglu4 ${ }^{4}$, Merve Ozgul5, Sevgi Kalayoglu Besisik6, Hakki Tanyeri ${ }^{7}$

${ }^{1}$ Associate Professor, Faculty of Dentistry, Department of Oral and Maxillofacial Surgery, Istanbul University, Fatih, Istanbul, Turkey. ${ }^{2}$ Faculty of Dentistry, Department of Oral and Maxillofacial Surgery, Istanbul University, Fatih, Istanbul, Turkey.

3Professor, Istanbul Faculty of Medicine, Department of Internal Disease, Istanbul University, Fatih, Istanbul, Turkey.

${ }_{4}^{4}$ Associate Professor, Faculty of Dentistry, Department of Oral and Maxillofacial Surgery, Istanbul University, Fatih, Istanbul, Turkey.

5 Faculty of Dentistry, Department of Oral and Maxillofacial Surgery, Istanbul University, Fatih, Istanbul, Turkey.

${ }^{6}$ Professor, Istanbul Faculty of Medicine, Department of Internal Disease, Istanbul University, Fatih, Istanbul, Turkey.

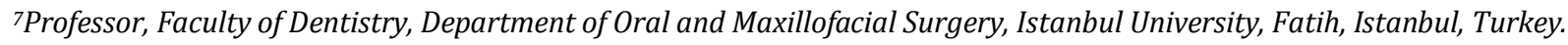

\section{ABSTRACT}

\section{BACKGROUND}

Recurrent Aphthous Stomatitis (RAS) is one of the most common oral mucosal diseases, characterised by recurrent painful mucosal ulcers. The aim of this study was to evaluate the prevalence of RAS in patients with hematinic deficiencies (HD).

\section{MATERIALS AND METHODS}

This cross-sectional study consisted of 201 patients with at least one HD parameter including iron, ferritin, folic acid, vitamin B12 and/or related complete blood count changes. The control group consisted of 200 healthy subjects, visited our clinic for dental check-ups.

\section{RESULTS}

There was no statistically significant difference between study and control groups in terms of RAS presence $(16.4 \%, 13.5 \%$; $>$ > 0.05). However, RAS history was present in 11 (27.5\%) of 40 patients with vitamin B12 deficiency (p=0.03), which was statistically significant.

\section{CONCLUSION}

Previous studies indicate HD may cause RAS and all these studies investigated the HD presence in patients with RAS. In order to assess whether HD is a cause of RAS or not, the presence of RAS in patients with HD should be taken into account. This study is the first study designed to assess the relationship between the two clinical conditions in this manner. There was no statistically significant relationship between RAS and serum iron, folic acid deficiency and anaemia, but only with B12 deficiency.

\section{KEYWORDS}

Hematinic Deficiency, Recurrent Aphthous Stomatitis.

HOW TO CITE THIS ARTICLE: Koray M, Ofluoglu D, Erden S, et al. The prevalence of recurrent aphthous stomatitis in patients with hematinic deficiency. J. Evolution Med. Dent. Sci. 2016;5(85):6310-6312, DOI: 10.14260/jemds/2016/1425

\section{BACKGROUND}

Recurrent Aphthous Stomatitis (RAS) is one of the most common oral mucosal diseases, characterised by recurrent painful mucosal ulcers which cause increased salivation and pain during eating, drinking and speaking. RAS affects approximately $20 \%$ of the general population, but the incidence ranges from $5 \%$ to $60 \%$ in different ethnical or socioeconomic groups. ${ }^{1}$

RAS is generally a topic of general medical practice, dental practice, internal medicine and dermatology. In spite of many investigations and studies, the aetiology of the RAS still remains unclear. Predisposing factors such as Hematinic Deficiencies (HD), heredity, immune dysregulation, certain

Financial or Other, Competing Interest: None.

Submission 03-10-2016, Peer Review 16-10-2016,

Acceptance 18-10-2016, Published 21-10-2016.

Corresponding Author:

Dr. Meltem Koray,

Associate Professor

Istanbul University, Faculty of Dentistry,

Department of Oral and Maxillofacial Surgery,

34093, Fatih Istanbul, Turkey.

E-mail:mkoray@istanbul.edu.tr

DOI: $10.14260 / j e m d s / 2016 / 1425$

\section{(c) (i) $\ominus$}

foods, drugs, stress, local trauma, hormonal disturbances, infections, smoking habits and poor oral hygiene may play a role in onset of RAS as well as preventing the disease. . $^{2-7}$

The role and prevalence of HD such as iron, folic acid and vitamin B12 deficiencies in the aetiopathogenesis of RAS are not well known and many conflicting results have been reported in the literature.4,5,7-10 Wray et al 8,11 reported increased incidence of HD in RAS patients and suggested haematological screening in such patients. While many researchers supported this relationship, ${ }^{5,9-13}$ conflicting studies exist as well. $4,14,15$ Olson et al 14 pointed out that serum deficiencies do not play a significant role in the aetiology of RAS. Koybasi et al ${ }^{4}$ reported a significant correlation between RAS and vitamin B12 deficiency, while there is no direct association between serum iron, ferritin and haemoglobin levels and RAS.

In the majority of the studies investigating the relationship between RAS and HD, patients with RAS diagnosis were analysed for HD and the results were compared with healthy subjects who had no RAS history. However, if HD is considered a cause of RAS, then the analysis should be made by investigating the RAS prevalence in patients with HD, not investigating the HD prevalence in patients with RAS. Based on the reported high prevalence of 
HD in RAS patients, we expected to find a high RAS incidence in patients with HD when compared with the normal population in terms of HD. We evaluated the RAS incidence in patients with HD and compared it with healthy control subjects.

\section{METHODS}

The study was designed as a cross-sectional study. The study group consisted of 201 patients only experiencing HD (188 Females, 13 Males, mean age 41.59 \pm 14.83 , range: 15-84). Patients were randomly selected from a group of patients who were referred to the outpatient clinic between August 2012 and January 2013.

Patients with a deficiency in at least one of the following hematinic parameters, serum iron, ferritin, folic acid, vitamin $\mathrm{B} 12$ and haemoglobin $(\mathrm{Hb})$ were included in the study. Blood samples were obtained between 09:00 am - 10:00 am. The control group consisted of 200 age-sex matched healthy control subjects (187 females, 13 males; mean age: 41.92 \pm 15.18 , range: $15-84$ ) without $\mathrm{HD}$ and systemic disorders who were referred to our clinic for dental checkups. Patients and subjects with any systemic disorder using any pharmacological therapies and/or drugs containing iron or vitamins, patients with Behcet's disease, coeliac disease and other gastrointestinal or dermatological diseases in which RAS is part of their clinical manifestation were excluded from the study.

A detailed medical history was recorded and intraoral clinical examination was performed on all patients and healthy subjects within the same day by two experienced surgeons. The RAS diagnosis was made based on medical history and clinical examination. Patients having at least one aphthous ulcer, defined as round or oval ulcers with a graywhite pseudomembrane and an erythematous halo less than $5 \mathrm{~mm}$ in diameter were considered as RAS positive. 6

Biochemical analyses were carried out with a Roche Hitachi E170 modular system, vitamin B12 was measured with Architect i2000 and i4000, and total blood count was analysed with the Cell Dyn-3700 from Abbott. The reference values for the parameters are as follows: $60-175 \mu / \mathrm{dL}$ for iron, 30-400 ng/mlt (Male) and 13-150 ng/mlt (Female) for ferritin, 3.1-17.5 ng/mlt for folic acid, $200-663 \mathrm{pg} / \mathrm{mL}$ for vitamin B12 and 13-15.5 g/dL (Male) and 12-15.5 g/dL (Female) for $\mathrm{Hb}$. All haematological analyses were evaluated by two haematologists.

All patients signed informed consent form and the procedures performed in this study involving human participants were in accordance with the ethical standards of the Declaration of Helsinki and approved by Istanbul University, Istanbul Medical Faculty Ethics Committee (Project No: 2013/1734). Data were analysed by using SPSS 20.0 statistic program (SPSS Inc., Chicago, IL, USA). Chisquare tests were used to evaluate the relationships between HD and RAS. Independent t-test was used to determine the means of samples. P value $\leq 0.05$ was considered statistically significant.

\section{RESULTS}

There was no statistically significant difference between study and control groups in terms of RAS ( $p>0.05)(16.4 \%$ and $13.5 \%$, respectively). Mean age was $41.93 \pm 15.71$ and $39.25 \pm 13.51$ in patients with and without RAS in the study group, respectively. In the control group, mean age was $40.58 \pm 14.29$ and $41.03 \pm 16.08$ in patients with and without RAS, respectively. There was no statistically significant difference between groups in terms of age $(p>0.05)$.

A great majority of the patients $(n=172,85.5 \%)$ in the study group had deficiencies in at least two hematinic parameters. Twenty-three of 146 patients with iron deficiency had an RAS history; however, there was no statistically significant relationship between iron levels and RAS $\left(x^{2}=0.17 ; p=0.17\right)$. Out of 40 patients $(27.5 \%)$ with $B 12$ deficiency, RAS was observed in 11 patients and the relationship between B12 deficiency and RAS was statistically significant $\left(\mathrm{x}^{2}=4.46 ; \mathrm{p}=0.03\right)$. RAS was observed in 22 patients out of 157 patients with anaemia and the relationship was not statistically significant $\left(x^{2}=3.02 ; p=0.08\right)$ (Table 1-2).

\begin{tabular}{|c|c|}
\hline Biochemical Analysis & Deficiency was Seen (n, \%) \\
\hline Iron Deficiency & $146(72.6 \%)$ \\
\hline Ferritin Deficiency & $125(62.2 \%)$ \\
\hline Vitamin B12 Deficiency & $40(19.9 \%)$ \\
\hline Hb Deficiency & $157(78.1 \%)$ \\
\hline \multicolumn{2}{|c|}{$\begin{array}{l}\text { Table 1. Hematinic Deficiencies of the Study } \\
\text { Group (n=201, Multiple HD was Included) }\end{array}$} \\
\hline
\end{tabular}

\begin{tabular}{|c|c|c|}
\hline Deficiencies & Patients with RAS (n, \%) & P-value \\
\hline $\begin{array}{l}\text { Iron Deficiency } \\
\qquad(\mathrm{n}=146)\end{array}$ & $23(15.8 \%)$ & $\mathrm{p}=0.17$ \\
\hline Ferritin Deficiency $(n=125)$ & $19(15.2 \%)$ & $\mathrm{p}=0.55$ \\
\hline $\begin{array}{c}\text { Vitamin B12 } \\
\text { Deficiency }(n=40)\end{array}$ & $11(27.5 \%)$ & $\mathrm{p}=0.03^{*}$ \\
\hline Hb Deficiency $(n=157)$ & $22(14 \%)$ & $\mathrm{p}=0.08$ \\
\hline \multicolumn{3}{|c|}{$\begin{array}{c}\text { Table 2. RAS Prevalence in HD Patients } \\
\text { (n=201, Multiple HD was Included) }\end{array}$} \\
\hline
\end{tabular}

Chi-square test was used ${ }^{*} \mathrm{p}<0.05$.

\section{DISCUSSION}

RAS is a common inflammatory ulcerative condition affecting the oral mucosa. It is an important condition that can be distressing and causes suffering and pain.16 Numerous studies have reported a high incidence (4-56, 2\%) of HD in patients with RAS.5,8,11,15-18 Iron, vitamin B12 and folic acid deficiencies were reported as predisposing factors in RAS in several studies. $4,5,8,10,11,16,18$

Porter et al ${ }^{18}$ have determined the frequency of anaemia and/or HD in RAS. They indicated that $11.6 \%$ of RAS patients had low iron storage when compared with $4.9 \%$ of control subjects and also indicated that neither patients nor control subjects had anaemia. Wray et al ${ }^{8}$ investigated 130 RAS patients for vitamin B12, folic acid and iron deficiencies and found that 23 patients (17.7\%) had HD. When compared to the control group, iron and folic acid deficiencies were found twice as frequently in RAS patients and vitamin B12 deficiency was five times more common in RAS patients. On the other hand, Rogers and Hutton ${ }^{19}$ studied 102 patients with RAS and found only $5.9 \%$ of patients to be anaemic.

Sun et al $^{13}$ reported a significant relationship between RAS and $\mathrm{Hb}$, iron, vitamin B12 and folic acid deficiencies. They suggested that these deficiencies may result in atrophy of the oral epithelium, which may contribute to or facilitate RAS development by breakdown of the oral epithelium. 
Lopez-Jornet et al 15 found a higher overall frequency $(14.14 \%)$ of HD in the RAS group versus the control group (6.39\%), but the difference was not statistically significant. Koybasi et $\mathrm{al}^{4}$ only found an association between vitamin B12 levels and RAS history. In clinical practice, vitamin B12 deficiency has multiple symptoms and signs in the oral mucosa. The most well-known finding is glossitis, which presents as a burning feeling in the mouth. ${ }^{20}$ Several studies have reported the role of vitamin B12 deficiency in RAS aetiology. $4,5,8,13$ It is also a well-known fact that vitamin B12 deficiency should be evaluated with folic acid level, which can be seen simultaneously. ${ }^{20,21}$ In the present study, a statistically significant difference was found between RAS and vitamin B12 deficiency, but no relationship was found between RAS and folic acid level.

Although, HD is proposed as a predisposing factor in the aetiology of RAS, all of the studies investigating the relationship between these two conditions are designed to determine whether or not HD is present in patients with RAS. However, studies also need to investigate the RAS prevalence in patients with HD in order to see if HD is a predisposing factor or not. Since there are conflicting results in the literature about the role of HD in RAS aetiology, we aimed to assess the relationship between these two conditions from another point of view. Therefore, we decided to investigate RAS prevalence in patients with HD. Based on existing literature on the relationship between these two conditions, we expected RAS incidence to be increased in patients with HD. But contrary to our expectations, RAS ratio was $16.4 \%$ in HD patients and $13.5 \%$ in control group subjects and this difference was not statistically significant ( $p>0.05$ ).

\section{CONCLUSION}

Without several studies investigating the relationship between HD and RAS, these results are still not confirmatory; there is no accepted consensus regarding HD screening protocol of RAS patients in the literature. This study aimed to investigate the relationship between RAS and HD from this point of view. Although, we did not find a statistically significant relationship between RAS and serum iron, ferritin, folic acid deficiencies and anaemia, we found a relationship between vitamin B12 deficiency and RAS history. The major limitation of this study was the non-uniform distribution of the patient population in terms of gender, which is composed of a high number of female patients. However, this limitation was an expected one, since HD is a more common condition in female patients. In conclusion, a definite statement with regard to the relation of RAS and HD could not yet be made yet, leaving the aetiology elusive. Taking account of many study results including this study it seems that HD screening, especially for vitamin B12 might be helpful in the management of RAS.

\section{REFERENCES}

1. Greenberg MS, Glick M. Ulcerative, vesicular, and bullous lesions. In: Burket's oral medicine diagnosis and treatment. 10th edn. Hamilton: BC Decker Inc 2003:63-65.

2. Jurge S, Kuffer R, Scully C, et al. Mucosal disease series. Number VI. Recurrent aphthous stomatitis. Oral Dis 2006;12(1):1-21.
3. Samet N, Laurent C, Susarla SM, et al. The effect of bee propolis on recurrent aphthous stomatitis: a pilot study. Clin Oral Investig 2007;11(2):143-7.

4. Koybasi S, Parlak AH, Serin E, et al. Recurrent aphthous stomatitis: investigation of possible etiologic factors. Am J Otolaryngol 2006;27(4):229-32.

5. Compilato D, Carroccio A, Calvino F, et al. Haematological deficiencies in patients with recurrent aphthosis. J Eur Acad Dermatol Venereol 2010;24(6): 667-73.

6. Scully C, Porter S. Oral mucosal disease: recurrent aphthous stomatitis. Br J Oral Maxillofac Surg 2008;46(3): 198-206.

7. Volkov I, Rudoy I, Froud T, et al. Effectiveness of vitamin B12 in treating recurrent aphthous stomatitis: a randomised, double-blind, placebo-controlled trial. J Am Board Fam Med 2009;22(1):9-16.

8. Wray D, Ferguson MM, Mason DK, et al. Recurrent aphthae: treatment with vitamin B12, folic acid and iron. Br Med J 1975;2(5969):490-3.

9. Khan NF, Saeed M, Chaudhary S, et al. Haematological parameters and recurrent aphthous stomatitis. J Coll Physicians Surg Pak 2013;23(2):124-7.

10. Kozlak ST, Walsh SJ, Lalla RV. Reduced dietary intake of vitamin B12 and folate in patients with recurrent aphthous stomatitis. J Oral Pathol Med 2010;39(5):420-3.

11. Wray D, Ferguson MM, Hutcheon WA, et al. Nutritional deficiencies in recurrent aphthae. J Oral Pathol 1978;7(6):418-23.

12. Burgan SZ, Sawair FA, Amarin ZO. Hematologic status in patients with recurrent aphthous stomatitis in Jordan. Saudi Med J 2006;27(3):381-4.

13. Sun A, Chen HM, Cheng SJ, et al. Significant association of deficiencies of hemoglobin, iron, vitamin B12, and folic acid and high homocysteine level with recurrent aphthous stomatitis. J Oral Pathol Med 2015;44(4):300-5.

14. Olson JA, Feinberg I, Silverman S, et al. Serum vitamin B12, folate, and iron levels in recurrent aphthous ulceration. Oral Surg Oral Med Oral Pathol 1982;54(5):517-20.

15. Lopez-Jornet P, Camacho-Alonso F, Martos N. Hematological study of patients with aphthous stomatitis. International Journal of Dermatology 2014;53(2):159-63.

16. Challacombe SJ, Scully C, Keevil B, et al. Serum ferritin in recurrent oral ulceration. J Oral Pathol 1983;12(4):290-9.

17. Safadi RA. Prevalence of recurrent aphthous ulceration in Jordanian dental patients. BMC Oral Health 2009;9:31.

18. Porter SR, Scully C, Flint S. Hematologic status in recurrent aphthous stomatitis compared with other oral diseases. Oral Surg Oral Med Oral Pathol 1988;66(1):41-4.

19. Rogers RS, Hutton KP. Screening for hematinic deficiencies in patients with recurrent aphthous stomatitis. Australas J Dermatol 1986;27(3):98-103.

20. Pontes HAR, Neto NC, Ferreira KB, et al. Oral manifestations of vitamin B12 deficiency: a case report. J Can Dent Assoc 2009;75(7):533-7.

21. Masri OA, Chalhoub JM, Sharara AI. Role of vitamins in gastrointestinal diseases. World J Gastroenterol 2015;21(17):5191-209. 